\title{
Optimum Threshold-Selection Relaying for Decode-and-Forward Cooperation Protocol
}

\author{
W. Pam Siriwongpairat, Thanongsak Himsoon, Weifeng $\mathrm{Su}^{\ddagger}$, and K. J. Ray Liu \\ Department of Electrical and Computer Engineering, University of Maryland, College Park, MD 20742 \\ $\ddagger$ Department of Electrical Engineering, State University of New York (SUNY) at Buffalo, Buffalo, NY 14260
}

\begin{abstract}
This paper proposes a threshold-selection relaying scheme, in which each relay decides whether to forward the source information by comparing the received signal power with a decision threshold. The challenging problem is to design the threshold such that the relay is able to forward only correctly decoded information. In this work, bit-error-rate (BER) performance analysis is provided for the proposed scheme with BPSK signals. We develop a BER upper bound which is tight over the entire range of signal-to-noise ratio values. Based on the established BER formulation, we jointly determine optimum decision threshold and power allocation. An interesting result is that the effect of optimum threshold dominates that of optimum power allocation, especially when the relay is close to the destination. For example, in case of equal power allocation, the proposed scheme with optimum threshold yields $10 \mathrm{~dB}$ performance improvement over the fixed relaying scheme without threshold at a BER of $10^{-2}$. If both the power allocation and the threshold are jointly optimized, then the performance of the proposed scheme can be further improved by $2 \mathrm{~dB}$.
\end{abstract}

\section{INTRODUCTION}

Cooperative diversity has recently emerged as a promising alternative to combat fading in wireless channels. The basic idea is that users or nodes in a wireless network share their information and transmit cooperatively as a virtual antenna array, thus providing diversity without the requirement of additional antennas at each node. Various cooperative protocols have been proposed for wireless networks [1]-[4]. In [3],[4], the authors proposed a two-user cooperation strategy for CDMA systems, in which pairs of users in the network are coupled to help each other and orthogonal codes are used to mitigate multiple access interference. In [1], the authors proposed different cooperation protocols including fixed and adaptive relaying protocol. In fixed relaying protocol, such as amplify-and-forward and decode-and-forward protocols, the relays always help forward the source information. With the amplify-and-forward protocol, the relays simply amplify and forward the information, whereas with the decode-andforward protocol, the relays decode the received information and then forward the decoded symbols to the destination. Since in fixed decode-and-forward protocol, the relay always forwards the decoded information to the destination, this protocol suffers performance loss if the relay cannot decode the transmitter's signal. To overcome the drawbacks of fixed relaying transmission, the authors in [1] also proposed adaptive relaying protocols including selection relaying and incremental relaying protocols. In selection relaying protocol, the relay forwards the information only if the amplitude of measured

\footnotetext{
${ }^{\dagger}$ This work was supported in part by U. S. Army Research Laboratory under Cooperative Agreement DAAD 190120011.
}

channel coefficient of source-relay link is larger than a certain threshold. With incremental relaying protocol, limited feedback from the destination is employed to indicate the success or failure of the direct transmission. Outage performance of these protocols is analyzed in [1]. Later in [5], the authors provided symbol-error-rate (SER) performance analysis and optimum power allocation for decode-and-forward cooperation systems with two users. The SER performance analysis of a class of multinode cooperative protocols was presented in [6].

In this paper, we propose a threshold-selection relaying scheme for decode-and-forward cooperation protocol in wireless networks. In the proposed scheme, each relay applies a threshold test on the received signal power from the source in order to decide whether to forward the source information. The challenge of the selection relaying protocol lies in how to decide the decision threshold. In this work, we analyze the bit-error-rate (BER) performance of the proposed scheme with binary phase shift keying (BPSK) signals. We provide an upper bound on the BER that is tight to the performance of the proposed scheme for every signal-to-noise ratio (SNR). Based on the tight BER upper bound, we are able to jointly determine optimum decision threshold and optimum power allocation. It turns out that the effect of optimum threshold dominates that of optimum power allocation, especially when the relay is located close to the destination. Simulation results show that with equal power allocation, the proposed selection relaying scheme with optimum threshold provides $10 \mathrm{~dB}$ performance gain over the fixed relaying scheme at a BER of $10^{-2}$. If optimum power allocation is also utilized, the performance of the proposed scheme with optimum threshold can be further improved by $2 \mathrm{~dB}$.

\section{SySTEM MODEL}

We present in this section a cooperation strategy for a wireless network, which can be a mobile ad hoc network or a cellular network. Each user (or node) in the network can be a source node that sends information to its destination, or it can be a relay node that helps transmit the other user's information. The cooperation strategy belongs to a class of selection relaying protocols [1], in which signal transmission involves two phases. In Phase 1, each source sends information to its destination, and the information is also received by other users in the network. In Phase 2, each relay compares the quality of the signal it receives in Phase 1 with a specific threshold. The relay decodes the received information and forward the decoded symbols to the destination only if the quality of the received signal is larger than the threshold; otherwise the relay does not send and remain idle. In both 


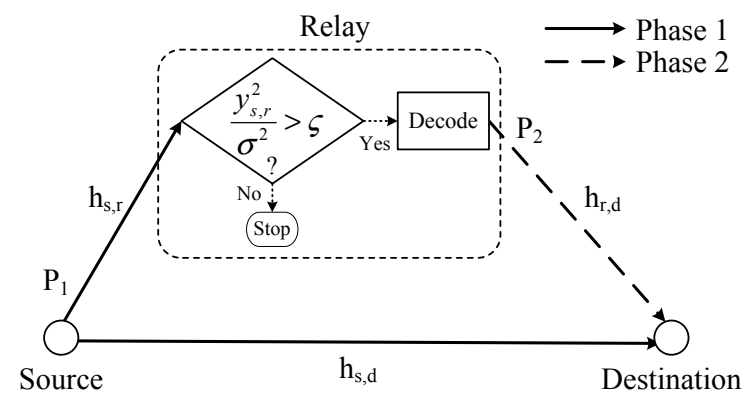

Fig. 1: Illustrations of the proposed selection decode-and-forward scheme with threshold $\zeta$.

phases, all users transmit signals through orthogonal channels by using TDMA, FDMA, or CDMA scheme [1]-[4].

The wireless link between any two nodes in the network is subject to fading and additive noise. Although the fading coefficients can be well estimated by the cooperating terminal, the additive noise is unknown to the receiver. In order to take into account both the effect of channel fading and the effect of additive noise, the quality of the received signal is measured from the amplitude of the received signal. Specifically, the relay decides whether to forward the signal it receives from the source by comparing the squared amplitude of the received signal, normalized by the average noise power, with a certain threshold. To better understand the cooperation concept, we focus on a two-user cooperation scheme in a real system with BPSK modulation, as illustrated in Figure 1.

In Phase 1, the source broadcasts its information to the destination and the relay. The received signals $y_{s, d}$ and $y_{s, r}$ at the destination and the relay respectively can be expressed as

$$
\begin{aligned}
& y_{s, d}=\sqrt{P_{1}} h_{s, d} x+z_{s, d}, \\
& y_{s, r}=\sqrt{P_{1}} h_{s, r} x+z_{s, r},
\end{aligned}
$$

where $x$ is the transmitted information symbol from BPSK modulation, $P_{1}$ is the transmitted power at the source, and $z_{s, d}$ and $z_{s, r}$ are additive noise. In (1) and (2), $h_{s, d}$ and $h_{s, r}$ are channel coefficients from the source to the destination and the relay, respectively. The channel coefficients are modeled as zero-mean, real Gaussian random variable with variances $\sigma_{s, d}^{2}$ and $\sigma_{s, r}^{2}$, respectively. The noise terms $z_{s, d}$ and $z_{s, r}$ are modeled as zero-mean real Gaussian random variables with variance $\sigma^{2}$.

In Phase 2, the relay decides whether to help forward the information it receives from the source by comparing $y_{s, r}^{2} / \sigma^{2}$ with a certain threshold $\zeta$. If the measured $y_{s, r}^{2} / \sigma^{2}$ falls below the threshold, then the relay does not forward the source's information. If the measured $y_{s, r}^{2} / \sigma^{2}$ lies above the threshold, then the relay decodes the received information and forwards the decoded symbol with power $P_{2}$ to the destination. Properly designed threshold enables the relay to efficiently make judgement on the correctness of the decoded symbols. Nevertheless, the condition that $y_{s, r}^{2} / \sigma^{2}$ is larger than the threshold $\zeta$ does not guarantee that the decoded symbol is correct. If this case happens, then the relay forwards wrong information symbol to the destination. Therefore, the received signal at the destination in Phase 2 can be modeled as

$$
y_{r, d}= \begin{cases}\sqrt{P_{2}} h_{r, d} \tilde{x}+z_{r, d}, & \text { if } y_{s, r}^{2} / \sigma^{2}>\zeta ; \\ z_{r, d}, & \text { if } y_{s, r}^{2} / \sigma^{2} \leq \zeta,\end{cases}
$$

where $\tilde{x}=x$ only if the relay decodes the transmitted signal correctly. Otherwise, $\tilde{x} \neq x$, e.g., $\tilde{x}=-x$ in case of BPSK signal. In (3), $h_{r, d}$ is the channel coefficient from the relay to the destination and $z_{r, d}$ is additive noise. The channel coefficient $h_{r, d}$ is modeled as a zero-mean real Gaussian random variable with variance $\sigma_{r, d}^{2}$, and the noise $z_{r, d}$ is modeled as a zero-mean real Gaussian random variable with variance $\sigma^{2}$.

We assume that the nodes are spatially well separated such that the channel fades for different propagation links are statistically independent. In addition, the channel coefficients are assumed known at the receiver, but not at the transmitter. The destination coherently combines the received signals from the source and the relay by using the maximum ratio combiner (MRC) [7]. The total transmitted power $P$ is fixed such that

$$
P_{1}+P_{2}=P .
$$

Note that the power savings in the case that the relay is idle is assumed negligible, since at high signal-to-noise ratio (SNR), the chance that $y_{s, r}^{2} / \sigma^{2}$ falls below the threshold is small, as will be shown later in the performance analysis.

\section{BER ANALYSIS}

In this section, we analyze the bit error rate (BER) performance for the proposed threshold-selection relaying scheme described in the previous section. First, we classify different scenarios that lead to different instantaneous SNR's of the combiner output at the destination. Next, the probability that each scenario occurs is determined. Then, we derive an average BER formulation of the cooperation system by taking into account all the possible scenarios.

\section{A. Classification of Different Scenarios}

With the knowledge of channel state information, the destination detect the transmitted symbols by jointly combining the received signals $y_{s, d}$ from the source and $y_{r, d}$ from the relay. The combined signal at the MRC detector can be given by [7]

$$
y=a_{1} y_{s, d}+a_{2} y_{r, d},
$$

where $a_{1}$ and $a_{2}$ are determined to maximize the SNR of the MRC output. Although it is possible that the relay forwards an incorrect symbol to the destination, this event occurs with small probability (especially at high SNR), and can be assumed negligible. Therefore, $a_{1}$ and $a_{2}$ can be specified as $a_{1}=\sqrt{P_{1}} h_{s, d} / \sigma^{2}$ and $a_{2}=\sqrt{\tilde{P}_{2}} h_{r, d} / \sigma^{2}$, where $\tilde{P}_{2}=P_{2}$ if $y_{s, r}^{2} / \sigma^{2}>\zeta$ and $\tilde{P}_{2}=0$ if $y_{s, r}^{2} / \sigma^{2} \leq \zeta$.

From (1)-(3) and (5), there are three scenarios that result in different SNR values of the combiner output. For subsequent analysis, we denote each scenario by $\Phi_{i}, i=1,2,3$, which is specified as follows:

$$
\begin{aligned}
& \Phi_{1} \triangleq\left\{y_{s, r}^{2} / \sigma^{2} \leq \zeta\right\}, \\
& \Phi_{2} \triangleq\left\{y_{s, r}^{2} / \sigma^{2}>\zeta, \tilde{x}=x\right\},
\end{aligned}
$$




$$
\Phi_{3} \triangleq\left\{y_{s, r}^{2} / \sigma^{2}>\zeta, \tilde{x} \neq x\right\} .
$$

The first scenario, $\Phi_{1}$, corresponds to the case when the measured signal quality of $y_{s, r}$ falls below the threshold $\left(y_{s, r}^{2} / \sigma^{2} \leq \zeta\right)$. In this case, the relay is idle, and the destination uses only the received signal $y_{s, d}$ from the source. Accordingly, the instantaneous SNR of the combiner output is the same as that of direct transmission, i.e.,

$$
\left.\gamma\right|_{\Phi_{1}}=\frac{P_{1} h_{s, d}^{2}}{\sigma^{2}}
$$

The scenario $\Phi_{2}$ corresponds to the case when the measured quality of $y_{s, r}$ is greater than the threshold, and the relay decodes the information correctly $(\tilde{x}=x)$. Under the scenario $\Phi_{2}$, the SNR of the combiner output is [7]

$$
\left.\gamma\right|_{\Phi_{2}}=\frac{P_{1} h_{s, d}^{2}+P_{2} h_{r, d}^{2}}{\sigma^{2}} .
$$

The last scenario, $\Phi_{3}$, also corresponds to the case that $y_{s, r}^{2} / \sigma^{2}$ lies above the threshold; however, the decoded symbol at the relay is incorrect.

\section{B. Probability of Occurrence}

For notational convenience, we denote $\mathrm{P}_{r}^{h}(A)$ as the conditional probability of event $A$, and denote $\mathrm{P}_{r}(A)=\mathrm{E}\left[\mathrm{P}_{r}^{h}(A)\right]$ as the average probability of $A$ over the channel distribution. From (6), the chance that the scenario $\Phi_{1}$ happens can be written as

$$
\mathrm{P}_{r}\left(\Phi_{1}\right)=\mathrm{P}_{r}\left(y_{s, r}^{2} \leq \zeta \sigma^{2}\right) .
$$

Since $y_{s, r}$ (see (2)) is a summation of two independent zero-mean Gaussian random variables, it is also Gaussian distributed with zero mean and variance

$$
q \triangleq P_{1} \sigma_{s, r}^{2}+\sigma^{2}
$$

Thus, $y_{s, r}^{2}$ is central chi-square distributed, and (11) can be obtained as [8]

$$
\mathrm{P}_{r}\left(\Phi_{1}\right)=\frac{1}{\sqrt{2 \pi q}} \int_{0}^{\zeta \sigma^{2}} \frac{1}{\sqrt{u}} \exp \left(-\frac{u}{2 q}\right) d u .
$$

Next, we determine the chance that the scenario $\Phi_{3}$ occurs, i.e., the chance of incorrect decoding at the relay while the received signal quality $y_{s, r}^{2} / \sigma^{2}$ is larger than the threshold. Based on the coherent detection, the decision variable at the relay can be given by

$$
h_{s, r} y_{s, r}=\sqrt{P_{1}} h_{s, r}^{2} x+h_{s, r} z_{s, r}
$$

Given a specific channel coefficient $h_{s, r}, h_{s, r} z_{s, r}$ is real Gaussian random variable with zero mean and variance $h_{s, r}^{2} \sigma^{2}$. Suppose $x=1$ is sent, then the decoded symbol is error if

$$
h_{s, r} z_{s, r} \leq-\sqrt{P_{1}} h_{s, r}^{2}
$$

Given $x=1$, the event that $y_{s, r}^{2} / \sigma^{2}>\zeta$ can be written as

$$
\left\{y_{s, r}^{2}>\zeta \sigma^{2}\right\}=\left\{\left|\sqrt{P_{1}} h_{s, r}+z_{s, r}\right|>\sigma \sqrt{\zeta}\right\}
$$

which is equivalent to

$$
\left\{z_{s, r} \leq-\sqrt{P_{1}} h_{s, r}-\sigma \sqrt{\zeta}\right\} \cup\left\{z_{s, r}>-\sqrt{P_{1}} h_{s, r}+\sigma \sqrt{\zeta}\right\}
$$

Multiplying each term in (17) with a real value $h_{s, r}$, we get

$$
\begin{aligned}
\left\{y_{s, r}^{2}>\zeta \sigma^{2}\right\}= & \left\{h_{s, r} z_{s, r} \leq-\sqrt{P_{1}} h_{s, r}^{2}-\sigma \sqrt{\zeta}\left|h_{s, r}\right|\right\} \\
& \cup\left\{h_{s, r} z_{s, r}>-\sqrt{P_{1}} h_{s, r}^{2}+\sigma \sqrt{\zeta}\left|h_{s, r}\right|\right\} .
\end{aligned}
$$

From (15) and (18), we can see that given $x=1$, the conditional bit error probability is simply the probability that $h_{s, r} z_{s, r} \leq-\sqrt{P_{1}} h_{s, r}^{2}-\sigma \sqrt{\zeta}\left|h_{s, r}\right|$. Assuming equiprobable symbols, the conditional probability that the scenario $\Phi_{3}$ occurs can be determined as

$$
\begin{aligned}
\mathrm{P}_{r}^{h}\left(\Phi_{3}\right) & =\int_{-\infty}^{-\sqrt{P_{1}} h_{s, r}^{2}-\left|h_{s, r}\right| \sqrt{\zeta \sigma^{2}}} p_{h_{s, r} z_{s, r}}(u) d u \\
& =Q\left(\frac{\sqrt{P_{1}}\left|h_{s, r}\right|}{\sigma}+\sqrt{\zeta}\right),
\end{aligned}
$$

where $p_{X}(x)$ denotes a probability density function (PDF) of $x$ and $Q(\cdot)$ is the Gaussian Q-function [8]. Using the fact that $Q(u)=\frac{1}{\pi} \int_{0}^{\pi / 2} \exp \left(-\frac{u^{2}}{2 \sin ^{2} \theta}\right) d \theta$ for $u \geq 0$ [9], we obtain the average probability $\mathrm{P}_{r}\left(\Phi_{3}\right)$ as

$$
\mathrm{P}_{r}\left(\Phi_{3}\right)=\frac{1}{\pi} \int_{0}^{\pi / 2} \mathrm{E}\left[\exp \left(-\frac{\left(\sqrt{P_{1}}\left|h_{s, r}\right| / \sigma+\sqrt{\zeta}\right)^{2}}{2 \sin ^{2} \theta}\right)\right] d \theta
$$

Since $h_{s, r}$ is zero-mean Gaussian distributed with variance $\sigma_{s, r}^{2},\left|h_{s, r}\right|$ is half Gaussian distributed with PDF $p_{\left|h_{s, r}\right|}(x)=$ $\frac{2}{\sqrt{2 \pi} \sigma_{s, r}} \exp \left(-x^{2} /\left(2 \sigma_{s, r}^{2}\right)\right)$. Averaging over the distribution of $\left|h_{s, r}\right|$, the chance that the scenario $\Phi_{3}$ occurs can be written as

$$
\mathrm{P}_{r}\left(\Phi_{3}\right)=\sqrt{\frac{2}{\pi^{3} \sigma_{s, r}^{2}}} \int_{0}^{\pi / 2} \int_{0}^{\infty} e^{-\frac{\left(\sqrt{P_{1}} v / \sigma+\sqrt{\zeta}\right)^{2}}{2 \sin ^{2} \theta}-\frac{v^{2}}{2 \sigma_{s, r}^{2}}} d v d \theta
$$

Now the chance that the scenario $\Phi_{2}$ occurs can be calculated as follows. First, observe from (7) and (8) that the scenarios $\Phi_{2}$ and $\Phi_{3}$ are disjoint. Thus, the chance that the scenario $\Phi_{2}$ happens can be determined from $\mathrm{P}_{r}\left(\Phi_{3}\right)$ as

$$
\mathrm{P}_{r}\left(\Phi_{2}\right)=\mathrm{P}_{r}\left(\Phi_{2} \cup \Phi_{3}\right)-\mathrm{P}_{r}\left(\Phi_{3}\right) .
$$

In (22), $\mathrm{P}_{r}\left(\Phi_{2} \cup \Phi_{3}\right)=\mathrm{P}_{r}\left(y_{s, r}^{2} / \sigma^{2}>\zeta\right)=1-\mathrm{P}_{r}\left(\Phi_{1}\right)$. Thus, $\mathrm{P}_{r}\left(\Phi_{2} \cup \Phi_{3}\right)$ can be obtained from (13) as

$$
\mathrm{P}_{r}\left(\Phi_{2} \cup \Phi_{3}\right)=\frac{1}{\sqrt{2 \pi q}} \int_{\zeta \sigma^{2}}^{\infty} \frac{1}{\sqrt{u}} \exp \left(-\frac{u}{2 q}\right) d u .
$$

Finally, the average probability that the scenario $\Phi_{2}$ happens can be determined from (21) and (23) as

$$
\begin{aligned}
\mathrm{P}_{r}\left(\Phi_{2}\right)= & \frac{1}{\sqrt{2 \pi q}} \int_{\zeta \sigma^{2}}^{\infty} \frac{1}{\sqrt{u}} e^{-u /(2 q)} d u-\sqrt{\frac{2}{\pi^{3} \sigma_{s, r}^{2}}} \\
& \times \int_{0}^{\pi / 2} \int_{0}^{\infty} e^{-\frac{\left(\sqrt{\left.P_{1} v / \sigma+\sqrt{\zeta}\right)^{2}}\right.}{2 \sin ^{2} \theta}-\frac{v^{2}}{2 \sigma_{s, r}^{2}}} d v d \theta .
\end{aligned}
$$

\section{Average BER analysis}

With an instantaneous SNR $\gamma$, the conditional BER of a BPSK system can be written as [9]

$$
\mathrm{P}_{e}^{h}=\Psi(\gamma) \triangleq \frac{1}{\pi} \int_{0}^{\pi / 2} \exp \left(-\frac{\gamma}{\sin ^{2} \theta}\right) d \theta
$$


According to the instantaneous SNR in (9), the conditional BER under the scenario $\Phi_{1}$ is

$$
\left.\mathrm{P}_{e}^{h}\right|_{\Phi_{1}}=\Psi\left(P_{1} h_{s, d}^{2} / \sigma^{2}\right)
$$

By averaging (26) over the channel coefficient $h_{s, d}$, we obtain the average BER under the scenario $\Phi_{1}$ :

$$
\begin{aligned}
\left.\mathrm{P}_{e}\right|_{\Phi_{1}} & =\frac{1}{\pi} \int_{0}^{\pi / 2} \mathrm{E}\left[\exp \left(-\frac{P_{1} h_{s, d}^{2}}{\sigma^{2} \sin ^{2} \theta}\right)\right] d \theta \\
& =\frac{1}{\pi} \int_{0}^{\pi / 2}\left(1-\frac{2 P_{1} \sigma_{s, d}^{2}}{\sigma^{2} \sin ^{2} \theta}\right)^{-1 / 2} d \theta,
\end{aligned}
$$

where the second equality follows from the moment generating function of the central chi-square random variable [8]. Similarly, the conditional BER under the scenario $\Phi_{2}$ can be obtained based on (10) as

$$
\left.\mathrm{P}_{e}^{h}\right|_{\Phi_{2}}=\Psi\left(\left(P_{1} h_{s, d}^{2}+P_{2} h_{r, d}^{2}\right) / \sigma^{2}\right) .
$$

With the assumption that $h_{s, d}$ and $h_{r, d}$ are independent, the average BER under the scenario $\Phi_{2}$ can be calculated in a similar manner to (27) as

$$
\left.\mathrm{P}_{e}\right|_{\Phi_{2}}=\frac{1}{\pi} \int_{0}^{\pi / 2}\left[\left(1-\frac{2 P_{1} \sigma_{s, d}^{2}}{\sigma^{2} \sin ^{2} \theta}\right)\left(1-\frac{2 P_{2} \sigma_{r, d}^{2}}{\sigma^{2} \sin ^{2} \theta}\right)\right]^{-1 / 2} d \theta .
$$

Under the scenario $\Phi_{3}$, it is difficult to obtain the conditional bit error probability, and the chance that this scenario happens is rare. For mathematical tractability and for better understanding of the system performance, we provide in what follows an upper bound on the BER performance. Specifically, the average BER under the scenario $\Phi_{3}$ is upper bounded by $1 / 2$, i.e.,

$$
\left.\mathrm{P}_{e}\right|_{\Phi_{3}} \leq 1 / 2
$$

Taking into account the three scenarios $\Phi_{i}$, we can express the conditional BER of the proposed scheme as

$$
\mathrm{P}_{e}^{h}=\left.\sum_{i=1}^{3} \mathrm{P}_{e}^{h}\right|_{\Phi_{i}} \mathrm{P}_{r}^{h}\left(\Phi_{i}\right)
$$

Observe from (6)-(8) that the scenarios $\Phi_{i}$ are independent of the channel coefficients $h_{s, d}$ and $h_{r, d}$. Therefore, averaging the above conditional BER over the fading channels $h_{s, d}, h_{s, r}$, and $h_{r, d}$, the average BER of the cooperation system can be upper bounded as

$$
\mathrm{P}_{e} \leq\left.\mathrm{P}_{e}\right|_{\Phi_{1}} \mathrm{P}_{r}\left(\Phi_{1}\right)+\left.\mathrm{P}_{e}\right|_{\Phi_{2}} \mathrm{P}_{r}\left(\Phi_{2}\right)+\mathrm{P}_{r}\left(\Phi_{3}\right) / 2 .
$$

After substituting the average BER under scenario 1 and 2 from, (27), (29), and the average probability that each scenario happens, (13), (24) and (21) into (31), we finally obtain the average BER of the cooperation scheme.

Figure 2 compares the BER formulation (31) and the simulated performance in case of BPSK modulation. For fair comparison, we plot average BER curves as functions of $P / \sigma^{2}$. The transmitted power is equally allocated, i.e., $P_{1}=P_{2}=P / 2$ and threshold is set at $\zeta=1$. We consider two channel environments: (i) $\sigma_{s, d}^{2}=\sigma_{s, r}^{2}=\sigma_{r, d}^{2}=1$; and (ii) $\sigma_{s, d}^{2}=\sigma_{r, d}^{2}=1$ and $\sigma_{s, r}^{2}=10$. Clearly, the theoretical results closely match the simulation results in all cases. This

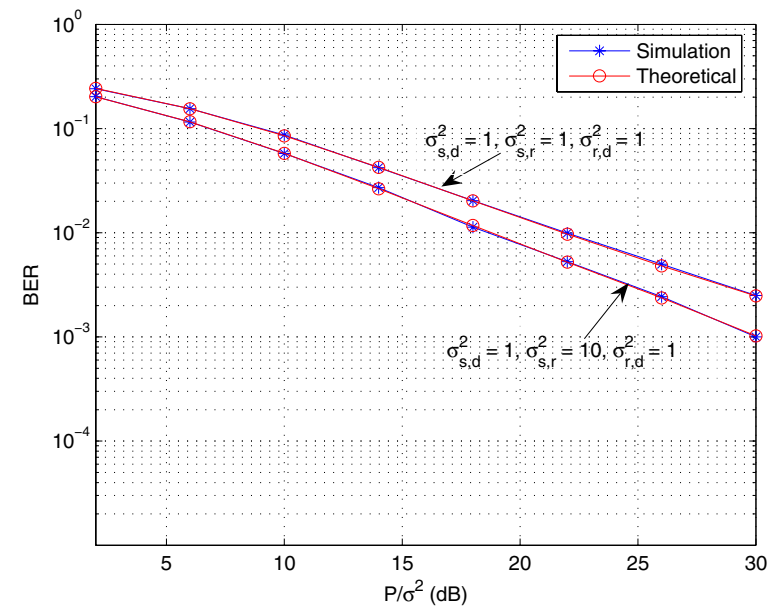

Fig. 2: BER performance versus $P / \sigma^{2}, P_{1}=P_{2}=0.5 P, \sigma^{2}=1$. We consider two environments: (i) $\sigma_{s, d}^{2}=\sigma_{s, r}^{2}=\sigma_{r, d}^{2}=1$; and (ii) $\sigma_{s, d}^{2}=\sigma_{r, d}^{2}=1, \sigma_{s, r}^{2}=10$.

confirms our expectation that the scenario $\Phi_{3}$ occurs with small probability. Hence, the effect of bounding the bit error probability under scenario $\Phi_{3}$ by 0.5 is insignificant.

\section{Optimum Decision Threshold AND Power ALLOCATION}

The average BER formulation derived in the previous section reveals that the choice of the power allocation and the threshold affects the performance of the cooperation scheme. In this section, we jointly determine an optimum decision threshold and an optimum power allocation for the proposed scheme based on the tight BER upper bound in (31). To simplify the notation, let us denote $r=P_{1} / P$ as the power ratio of the transmitted power at the source $\left(P_{1}\right)$ to the total power $(P)$. For a fixed total transmitted power $P_{1}+P_{2}=P$, we are going to jointly optimize the threshold $\zeta$ and the power ratio $r$ such that the tight BER upper bound in (31) is minimized. The optimization problem can be formulated as

$$
\min _{\zeta, r} P_{B E R}(\zeta, r),
$$

where $P_{B E R}(\zeta, r)$ represents the BER formulation in (31) with $P_{1}=r P$ and $P_{2}=(1-r) P$.

Figures 3-5 show the BER performance of the proposed selection decode-and-forward scheme under different power allocations and thresholds. The total transmitted power is fixed at $P=24 \mathrm{~dB}$ and the noise variance is equal to 1 . We consider a case of $\sigma_{s, d}^{2}=1, \sigma_{s, r}^{2}=1, \sigma_{r, d}^{2}=10$, e.g., the relay is located close to the destination. The BER formulation is plotted in Figure 3 as a function of power ratio and threshold, and its cross sections are shown in Figures 4 and 5 together with the simulated BER curves. Based on the BER plot in Figure 3, we obtain an optimum power allocation of $r=0.85$ and an optimum threshold of $\zeta=3$. Figure 4 compares the cross sectional curve of the theoretical BER with the simulated BER in case of optimum power ratio $r=0.85$. We can see that the theoretical BER is close to the simulated BER. When threshold is small, we can observe a performance gap 


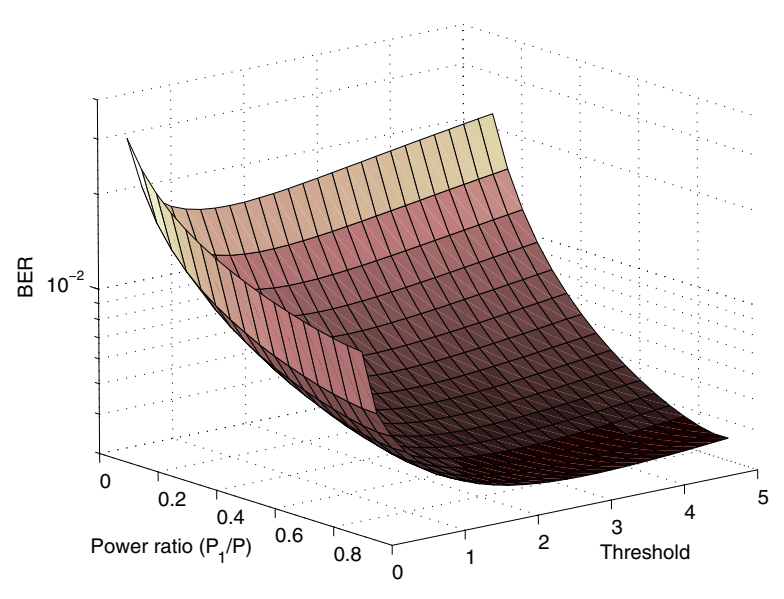

Fig. 3: Theoretical BER performance versus threshold and power ratio.

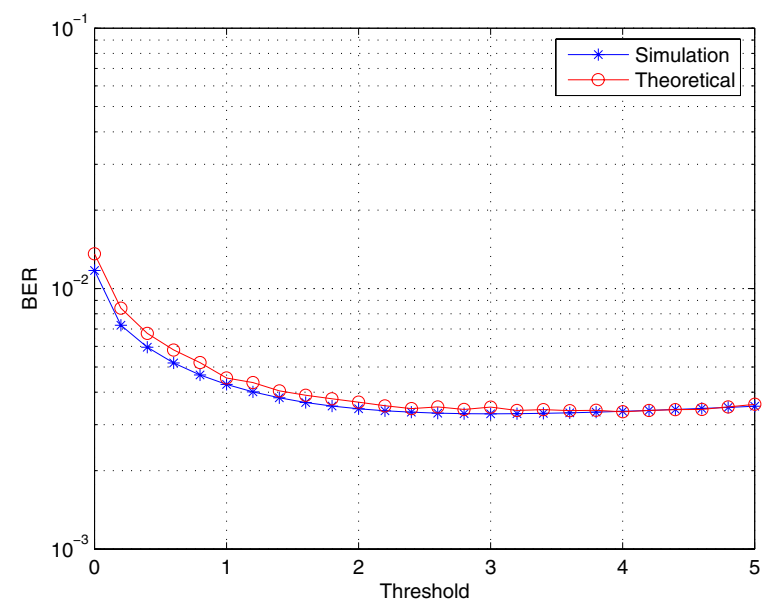

Fig. 4: BER performance versus threshold: power allocation is fixed at $P_{1}=0.85 P, P_{2}=0.15 P$.

between the simulated BER and the theoretical BER. This is due to the approximation on the bit error probability under the scenario $\Phi_{3}$. Furthermore, the performance significantly degrades as threshold decreases to zero. This is due to the fact that at small threshold, the relay tends to forward most of its received information which in turn increases the chance that incorrect symbols are sent to the destination. Figure 5 depicts the theoretical and simulated BER curves as functions of power allocation in case of the decision threshold $\zeta=3$. We can see that the power ratio of $r=0.85$ results in the optimum performance.

\section{Simulation Results}

We perform computer simulations of the decode-andforward cooperation system to illustrate the above theoretical analysis. In all simulations, we assume that the noise variance is $\sigma^{2}=1$, and the variance of the channel link between the source and the destination is $1\left(\sigma_{s, d}^{2}=1\right)$. We consider two channel conditions: (i) $\sigma_{s, r}^{2}=1$ and $\sigma_{r, d}^{2}=1$; and (ii) $\sigma_{s, r}^{2}=1$ and $\sigma_{r, d}^{2}=10$. For fair comparison, we present the average BER curves as functions of $P / \sigma^{2}$. The power allocation at the source node and relay node is fixed at $P_{1}+P_{2}=P$. BPSK modulation is used in all simulations.

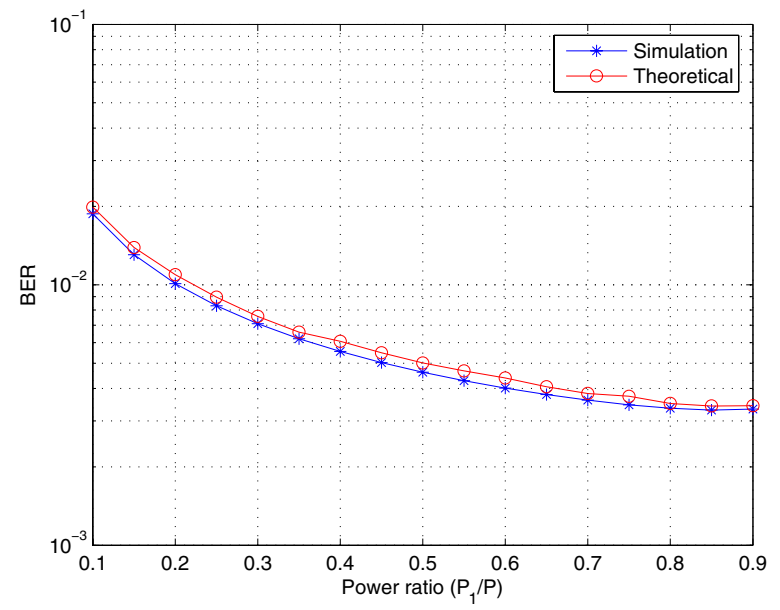

Fig. 5: BER performance versus power ratio: threshold is fixed at $\zeta$ $=3$.

In Figure 6, we compare the theoretical and simulated performance of the proposed scheme under the channel condition $\sigma_{s, r}^{2}=1$ and $\sigma_{r, d}^{2}=10$. We consider the case when transmitted power is optimally allocated at the source and the relay. From the BER performance in Figure 3, the optimum power allocation under this channel condition is $P_{1}=0.85 P$ and $P_{2}=0.15 P$. Figure 6 depicts the performance of the selection relaying scheme with optimum threshold, and that of the fixed relaying scheme (i.e. the threshold is $\zeta=0$; in other words, there is no threshold at the relay and the relay always forwards the information to the destination). We can see that the theoretical BER in (31) closely matches the simulation results in all cases. Moreover, selection relaying with the optimum threshold outperforms the fixed decode-andforward scheme by $7 \mathrm{~dB}$ at a BER of $10^{-2}$.

Figures 7 and 8 compare the simulated performance of the cooperation protocol with different power allocations and thresholds. Specifically, we plot the performance of (1) fixed relaying scheme with equal power allocation, (2) fixed relaying scheme with optimum power allocation, (3) selection relaying scheme with optimum threshold and equal power allocation, and (4) selection relaying scheme with optimum threshold and optimum power allocation.

Figure 7 depicts the performance under the channel condition $\sigma_{s, r}^{2}=1$ and $\sigma_{r, d}^{2}=1$. Based on the BER formulation in (31), we can obtain the optimum threshold of $\zeta=3$ and optimum power allocation of $P_{1}=0.75 P$ and $P_{2}=0.25 P$. We can see that the selection relaying with optimum threshold scheme achieves superior performance to that of fixed relaying scheme for any power allocation. The reason behind this is that a decoding error at the relay tends to result in an error at the destination. Hence, relaying all the received symbols leads to worse performance than forwarding only when the received signal is of high quality. Interestingly, the results in Figure 7 indicates that the effect of the threshold dominates that of the power allocation. For example, with equal power allocation, the fixed relaying scheme with optimum threshold achieves 5.5 $\mathrm{dB}$ performance improvement over the fixed relaying scheme. By also allocating power optimally, the performance gain of the fixed relaying scheme can be further improved to $6.5 \mathrm{~dB}$ 




Fig. 6: Comparison of simulation and theoretical formulation, assuming $\sigma_{s, r}^{2}=1$ and $\sigma_{r, d}^{2}=10$.

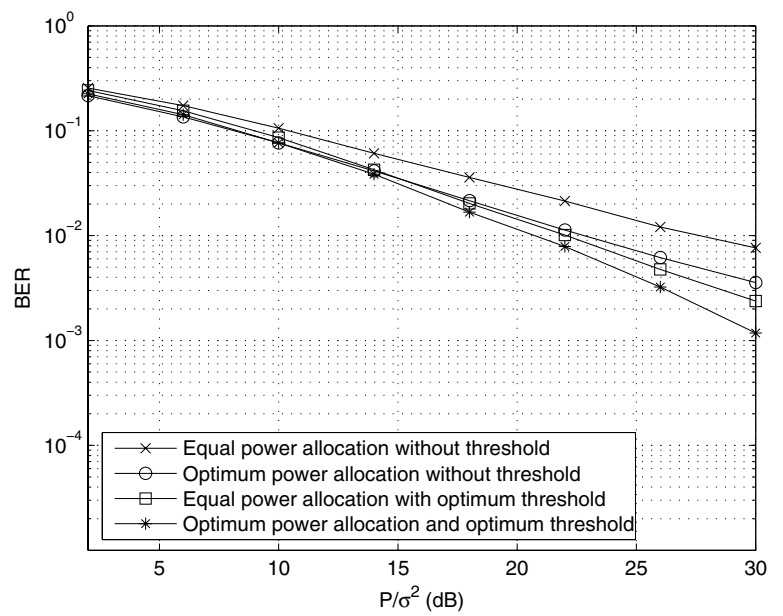

Fig. 7: Simulation of the proposed selection decode-and-forward scheme, assuming $\sigma_{s, r}^{2}=\sigma_{r, d}^{2}=1$.

at the BER of $10^{-2}$.

Figure 8 illustrates the simulated performance under the channel condition $\sigma_{s, r}^{2}=1$ and $\sigma_{r, d}^{2}=10$, which corresponds to the scenario when the relay is located close to the destination. Observe that the proposed selection relaying scheme with both optimum power allocation and optimum threshold yields the best performance over the entire SNR range. Moreover, it is clear that when the quality of relay-destination link is very good, the use of optimum threshold is more important than the use of optimum power allocation especially at high SNR. This is due to the fact that the relay-destination link is of high quality, so the received signal from the relay is of high SNR. If the relay forwards an incorrect symbol, then there is a good chance that the destination decodes the transmitter's signal incorrectly. As we can see from Figure 8, in the case of equal power allocation, the selection relaying scheme with optimum threshold achieves about $10 \mathrm{~dB}$ improvement over the fixed relaying scheme at a BER of $10^{-2}$. With optimum threshold, the performance difference between the selection relaying scheme with optimum power allocation and that with equal power allocation is only about $2 \mathrm{~dB}$ at the same BER.



Fig. 8: Simulation of the proposed selection decode-and-forward scheme, assuming $\sigma_{s, r}^{2}=1$ and $\sigma_{r, d}^{2}=10$.

\section{CONCLUSIONS}

In this paper, we propose a threshold-selection relaying scheme that adapts based on the received signal power at relay terminals. Bit error rate (BER) performance analysis is provided for real system with BPSK signals. Optimum threshold and optimum power allocation are jointly determine based on the established BER formulation. Both theoretical and simulation results reveal that the optimum threshold and optimum power allocation rely on the qualities of the channel links. When the quality of the relay-destination link is much larger than that of the other links, then the threshold is more important than the power allocation at high SNR. For example, with equal power allocation, the proposed scheme with optimum threshold results in $10 \mathrm{~dB}$ performance improvement gain over the fixed relaying scheme without threshold at a BER of $10^{-2}$. By also using the optimum power allocation, the performance of the proposed scheme is further improved by $2 \mathrm{~dB}$ at the same BER.

\section{REFERENCES}

[1] J. N. Laneman and G. W. Wornell, "Distributed space-time coded protocols for exploiting cooperative diversity in wireless networks," IEEE Trans. Inform. Theory, vol. 49, pp. 2415-2525, Oct. 2003.

[2] J. N. Laneman, D. N. C. Tse, and G. W. Wornell, "Cooperative diversity in wireless networks: efficient protocols and outage behavior," IEEE Trans. Inform. Theory, vol. 50, no. 12, pp. 3062-3080, Dec. 2004.

[3] A. Sendonaris, E. Erkip, and B. Aazhang, "User cooperation diversity, Part I: system description," IEEE Trans. on Commun., vol. 51, no. 11, pp. 1927-1938, Nov. 2003.

[4] A. Sendonaris, E. Erkip, and B. Aazhang, "User cooperation diversity, Part II: implementation aspects and performance analysis," IEEE Trans. on Commun., vol. 51, no. 11, pp. 1939-1948, Nov. 2003.

[5] W. Su, A. K. Sadek, and K. J. R. Liu, "SER performance analysis and optimum power allocation for decode-and-forward cooperation protocol in wireless networks," IEEE WCNC, vol. 2, pp. 984-989, Mar. 2005.

[6] A. K. Sadek, W. Su, and K. J. R. Liu, "Performance analysis for multinode decode-and-forward relaying in cooperative wireless networks," in Proc. IEEE Inter. Conf. on Acoustics, Speech, and Signal Proc., vol. 3, pp. 521-524, Mar. 2005.

[7] D. G. Brennan, "Linear diversity combining techniques," Proceedings of the IEEE, vol. 91, no. 2, pp.331-356, Feb. 2003.

[8] J. G. Proakis, Digital Communications, $4^{\text {th }}$ Ed., McGraw-Hill, New York, 2001.

[9] M. K. Simon and M. S. Alouini, Digital Communication over Fading Channels: A Unified Approach to Performance Analysis, John Wiley and Sons, New York, 2000 